\title{
The Power of Conventions: A Theory of Social Preferences ${ }^{1}$
}

\author{
Jing Li \\ Department of Economics \\ University of Pennsylvania \\ 3718 Locust Walk \\ Philadelphia, PA 19103 \\ E-mail: jing.li@econ.upenn.edu
}

November, 2005

${ }^{1}$ I am indebted to Bart Lipman and Larry Samuelson for invaluable guidance in conducting economics research in general and in writing this paper specifically. I thank Jim Andreoni, Pierpaolo Battigalli, Ming Li, Bill Sandholm, an associate editor and an anonymous referee for very useful comments. I also wish to thank fellow campers at the 2002 Russell Sage Behavioral Economics Camp for inspiring discussions and comments, especially Hanming Fang, David Laibson, George Lowenstein and Matthew Rabin. 


\begin{abstract}
Real life evidence suggests people act as if they care about others' welfare as well as their own, i.e. have "social preferences." I propose a model for such phenomena by incorporating players' preferences for social implications of their actions, determined by exogenous "conventions," in addition to the material consequences of the actions. A convention induces rankings over one's feasible actions, conditional on one's belief about the opponent's action. I construct games with conventions using the psychological games framework developed in Geanakoplos, Pearce and Stacchetti (1989). With a notion of distributional convention that incorporates the efficiency and fairness considerations, I show that equilibrium behavior in games with such conventions reflects social preferences. The model yields tight and testable predictions consistent with a large body of experimental results, is parsimonious and is suggestive of further studies, both experimentally and theoretically.
\end{abstract}

Keywords: convention, social preferences, coordination, fairness, psychological games 


\section{Introduction}

Social preferences refer to the phenomena that people seem to care about certain "social" goals, such as well-beings of other individuals, or a "fair" allocation among members in society, in addition to their own material benefits. The evidence is ample; Camerer(2003), Kahneman and Tversky (2000) and Sobel (2004) all contain extensive account of both real life examples and experimental results.

Depending on the fine details of the environment, social preferences exhibit many patterns: sometimes people reciprocate - reward kindness and punish unkindness; sometimes people show unmotivated altruism; sometimes people act in the entire group's interest, even if it hurts some individuals in the group. The following experimental results are illustrative of the variety of the patterns of social preferences.

1. In an experiment of the dictator game 1 pubjects choose between pairs of (self, other) allocations. About $50 \%$ of the subjects choose $(375,750)$ over $(400,400)$ (Charness and Rabin 2002).

2. Subjects first play a dictator game, choosing between (self, other) allocations of $(\$ 10, \$ 10)$ and $(\$ 18, \$ 2) 2^{2}$ Then some choices were randomly selected and realized. Finally, those subjects whose decisions were not realized were given the choice of evenly splitting $\$ 12$ with a person whose first offer was $(18,2)$ or evenly splitting $\$ 10$ with a person whose first offer was $(10,10)$. The one who was not chosen for the interaction receives 0 . About $74 \%$ of the subjects chose the latter (Kahneman, Knetsch and Thaler 1986).

3. Two players sequentially make private contributions to a public good, which is supplied either at the maximum of the two contributions (the best-shot game) or at the sum of them (the summation game). The first-mover has a smaller marginal-willingness-to-pay than the second-mover $3^{3}$ Subjects behave very differently in experiments of these two games: the first-mover typically does free ride in the best-shot game, but not in the summation game; in addition, when the first-mover contributes 0 , the second-mover responds by contributing 0 almost three times more often in the summation game than in the best-shot game (Andreoni, Brown and Vesterlund 2002).

4. The ultimatum game is another famous example where theoretical prediction

\footnotetext{
${ }^{1}$ The dictator game is a one-player game in which the player allocates a sum of money between himself/herself and another inactive player. Obviously, traditional game theory predicts the player takes everything himself.

${ }^{2}$ Consistent with the previous result, in this experiment 122 out of 161 subjects chose $(10,10)$ over $(18,2)$.

${ }^{3}$ Subgame perfect equilibrium predicts the same outcome in both games: the first-mover free rides and the second-mover provides the entire public good.
} 
fails 4 In laboratory experiments, it is rarely observed that the proposer demands the entire sum, and offers of $20-30 \%$ are frequently rejected. Offers of $50 / 50$ split are observed in all experiments, oftentimes being the mode. With stakes between $\$ 5$ and $\$ 20$ and as high as $\$ 100$, the average offer is around $40 \%$ of the sum. Moreover, the rejection rate seems to depend on possible offers the proposer did not make. For instance, when the proposer chooses between offering $20 \%$ or $75 \%$, an offer of $20 \%$ is rejected $33 \%$ of the time; however, when the proposer's choice set is changed to $(20 \%, 87.5 \%)$, the rejection rate for an offer of 20\% drops to 16\%. (Brandts and Sola 2001, Camerer and Thaler 1995, Charness and Rabin 2002, Thaler 1988).

It turns out to be a challenging task to explain all these complex patterns in a parsimonious model. The existing literature on social preferences includes two main classes of models, the distributional preferences models and the reciprocal preferences models.

Distributional preferences models assume players have preferences over final payoff allocations. For example, Fehr and Schmidt (1999) and Bolton and Ockenfels (2000) explain the ultimatum game results by assuming that players dislike inequality in final payoff allocations. However, these models cannot explain why players prefer an unequal payoff allocation to their own disadvantage as in Example 1. Altruism and social welfare models along the line of Andreoni (1990) and Andreoni and Miller (2002) assume players prefer a higher payoff for the opponents or the entire group of players in the game. These models can explain self-sacrificing behavior as in Examples 1 and 3, but cannot explain Pareto damaging behavior such as rejecting low offers in ultimatum games.

In fact, Example 3 clearly indicates that players' preferences over final payoff allocations alone cannot explain social preferences. When the first-mover contributes nothing, the set of payoff allocations the second-mover can generate is exactly the same in the two public-good games. Yet the second-mover makes systematically different choices. There must be something other than final payoff allocations that enters players' considerations.

In a seminal paper (1993), Rabin argues that it is reciprocity that makes the difference. Rabin assumes social preferences are driven by players' kindness towards each other: if a player believes the opponent's action is motivated by kindness toward him, he then prefers to react kindly; and vice versa. This model successfully accounts for retaliatory and altruistic behavior. Such reciprocal preferences models are intuitively appealing and further explored in Falk and Fischbacher (2000) and Dufwenberg and Kirchsteiger (2004), among others.

\footnotetext{
${ }^{4}$ In the ultimatum game, two players split a sum of money. The proposer moves first and makes an offer to the responder. If the responder accepts the offer then the money is divided as such. If the responder rejects it, then both players get nothing. In all subgame perfect equilibria of this game, the proposer makes an offer of no greater than the minimum share he can offer, and the responder accepts whatever she is offered.
} 
Rabin (1993) is a zero-parameter model. Players strictly prefer either the kindest action or the meanest action, depending on their beliefs about the opponent. Such pure reciprocity does not explain unmotivated altruistic behavior (Example 1), or why subjects would punish, at their own cost, somebody who is mean to another person (Example 2). In addition, the simple split-the-difference fairness notion often fails to capture the context of the game. For instance, according to this fairness function, in Example 4, offering 20\% would be strictly fairer in the first game than in the second game, if the proposer can offer 0. But then there should be less rejections in the first game than in the second, inconsistent with the experimental results.

One solution is to write the missing components into the utility function and adding parameters. In fact, in the appendix, Rabin (1993) suggests that, to have unmotivated altruism in the model, one could add a parameter to capture the relative strength of such concerns in comparison to that of reciprocity.5 The unsatisfactory performance of the naive fairness function could be due to its lack of an objective criterion reflecting social concerns as suggested in the distributional preferences models.

Charness and Rabin (2002) suggests a comprehensive model along this line. Their model uses six parameters to summarize how players weigh social preferences in their total preferences, how they weigh fairness in comparison to efficiency, and how they punish those opponents who they believe to fail to be sufficiently concerned about other people according to some social standard. The model formalizes the most important heuristic patterns of social preferences observed in experiments. In particular, it combines distributional preferences with reciprocal preferences. The model fits important experimental results in the literature. However, with so many parameters, the model is rather unrestrictive in making interpretations and predictions, while at the same time is too restrictive in that it does not allow for heterogeneity across players or any other forms of social preferences.

In this paper, I explore an alternative perspective to social preferences. In each of the above examples, the players seem to share some normative standpoint of what each of them "ought to" do given what could be done. I refer to this normative standpoint "the convention." Players prefer to conform to the convention, and prefer their opponents to conform to the convention as well. In the dictator game and the ultimatum game, it is conventional for the proposer to share the money with the opponent evenly. In the public-good game, it is conventional for players to contribute as long as they benefit from the public good and as long as their contributions count. Under common knowledge of these conventions, subjects are willing to give up some material benefits in order to conform to the convention (Experiment 1), to choose an opponent who conforms to the convention (Experiment 2 and the best-shot game in Experiment 3), and when having to interact with an opponent who has the opportunity to conform to the convention but chooses not to, to refuse to conform to the

\footnotetext{
${ }^{5}$ I thank an associate editor for bringing to my attention the discussion about more general utility functions in the appendix of Rabin (1993).
} 
convention themselves (the summation game in Experiment 3 and Experiment 4).

A notable departure of the above story from the distributional preferences models is that players do not care about others' welfare per se. Rather, they only try to conform to conventions, which may incorporate some received notions about how the resources should be allocated among players. On the other hand, this explanation is also subtly different from reciprocal preferences models in that players do not care about the opponents' intentions towards themselves, instead, they care about the opponents' intentions towards the convention: how much the opponents conform to the convention comparing to themselves.

I assume players receive payoffs from the social implications of their actions according to the convention, and the payoffs come from two additively separable components: conformity effects - players prefer to conform to the convention, and interaction effects - players prefer their opponents to conform to the convention to a degree at least as much as themselves. The total payoffs are the weighted average of the material payoffs and such social payoffs. The weight is interpreted as the salience of the convention one perceives in a game, which can be heterogeneous across players.

Intuitively, a convention induces a ranking over all possible actions in terms of their "appropriateness" or the degree of "right and wrong." The higher an action is ranked, the more desirable it is in terms of its social implications. Whether an action is appropriate depends on what appears to be the relevant context, which in turn depends on the player's feasible alternatives and beliefs about the opponent's action. Therefore, I model conventions as rankings of all actions conditional on the player's belief about the opponent's action. Assuming common knowledge of the convention and payoffs, ${ }^{6}$ using the psychological games framework developed in Geanakoplos, Pearce and Stacchetti (1989) (henceforth GPS), I construct games incorporating conventions for two-person normal-form games.

Conventions are exogenous in this model. In other words, the convention is part of the definition in the psychological game. In principle, conventions could reflect political ideal, religion, tradition etc., and do not necessarily depend on payoffs. The same material game can be associated with different conventions, depending on the contexts of the game. In economic context, it seems the most relevant conventions are criteria regarding allocations of the payoffs. Thus, I am most interested in distributional convention, which is based on payoff allocations and reflects some social standard based on efficiency and fairness criteria. Social preferences emerge naturally in equilibrium of games with distributional convention. With only one parameter summarizing the (heterogeneous) attitude towards conventions across players

\footnotetext{
${ }^{6}$ That the convention is common knowledge is a critical assumption for this model, because for players to take into account the social implications of each other's actions, the payoffs derived from the convention have to be common knowledge. On the other hand, that the convention induces a unique ranking over one's strategy space is a simplifying assumption that rules out uncertainty of payoffs. It should be possible to relax the latter assumption but it is less obvious when it comes to the former. For more discussions along this line, see sections 4 .
} 
and two parameters summarizing the distributional convention, the model generates tight predictions consistent with a large body of experimental results. The separation of conventions and players' attitudes towards conventions makes it possible to isolate the effects of changes in conventions and heterogeneity across players on equilibrium behavior, which is suggestive for further experimental study. The general model could incorporate a wide range of social effects. In the class of games I am most interested in, namely games with distributional conventions, the model is comparable to Charness and Rabin (2002), but with a more flexible structure, less parameters and heterogeneous players.

The paper proceeds as the follows. Section 2 presents the basic model. Section 3 focuses on models with distributional convention. Two examples are discussed at length: the symmetric two-by-two games (including the prisoner's dilemma game, the pure coordination game and the chicken game) and the public-good games (including the summation game and the best-shot game). Section 4 concludes. Proofs not found in the text are collected in the appendix.

\section{A Model}

Fix a two-person normal form game. Let $S_{i}$ and $\Sigma_{i}, i=1,2$ denote player $i$ 's finite set of pure strategies and set of mixed strategies respectively. Following Rabin (1993), I use $b$ and $c$ to denote the first and second-order beliefs respectively. That is, $b_{i} \in \Sigma_{i}$ is $j$ 's belief about $i$ 's strategy, and $c_{i} \in \Sigma_{i}$ is $i$ 's belief about $j$ 's belief about $i$ 's strategy ${ }^{7}$ Let $\pi_{i}(\sigma)$ be the material payoff player $i$ receives if strategy profile $\sigma=\left(\sigma_{1}, \sigma_{2}\right) \in$ $\Sigma_{1} \times \Sigma_{2}$ is played.

Suppose besides the material payoffs, players also care about some exogenous convention that is applicable in the particular context of the game. To incorporate such considerations, I first expand the game to include a description of the convention players have in mind.

There are two subtleties concerning conventions. First, for any action, there is typically no absolute social implication attached to it. Rather, an action may be regarded as right or wrong, depending on what else one could have done 8 For example, a doctor who recommends an expensive physical therapy is doing the right thing if there is no better treatment available, while is not if he is aware of an effective and cheap new drug but chooses not to mention it to the patient. Secondly, in strategic situations, the right thing to do depends on what the opponent is doing. For an obvious example, compare the different driving conventions in the U.K. and in the U.S. Therefore, I model the convention as belief-dependent rankings over one's

\footnotetext{
${ }^{7}$ I assume all beliefs are degenerate. See footnote 10 for a related discussion.

${ }^{8}$ Andreoni, Brown and Vesterlund (2002) document experimental results indicating the relevance of unchosen but available alternatives in subjects' fairness consideration. Also see Brandts and Sola (2001), Falk, Fehr and Fishbacher (2000).
} 
strategy space, and normalize them to the unit interval.

Definition 1 Fix a two-person normal form game $G$. A convention of $G$ is a function $\omega: \Sigma_{1} \times \Sigma_{2} \rightarrow[0,1]^{2}$ such that, for $i, j=1,2$,

1. for all $b_{j} \in S_{j}$, either $\omega_{i}\left(s_{i} ; b_{j}\right)=1$ for all $s_{i} \in S_{i} ;$ or $\max _{s_{i} \in S_{i}} \omega_{i}\left(s_{i} ; b_{j}\right)=$ 1 and $\min _{s_{i} \in S_{i}} \omega_{i}\left(s_{i} ; b_{j}\right)=0$;

2. for all $\sigma_{i} \in \Sigma_{i}, b_{j} \in \Sigma_{j}, \omega_{i}\left(\sigma_{i} ; b_{j}\right)=\sum_{s_{i}} \sum_{s_{j}} \sigma_{i}\left(s_{i}\right) b_{j}\left(s_{j}\right) \omega_{i}\left(s_{i} ; s_{j}\right)$.

Condition 1 says $\omega_{i}$ ranks $i$ 's pure actions conditional on $i$ 's belief of $j$ 's action and the ranking is normalized to the unit interval, so that dependence on $S_{i}$ is suppressed. The number $\omega_{i}\left(s_{i} ; b_{j}\right)$ is referred to as the social index of $i$ taking the action $s_{i}$ given his set of strategies $S_{i}$ and belief $b_{j}$. For example, in the driving convention example, under the belief that the opponent drives on the left side of the road (in the U.K.), the action "driving at the left side" is attached a social index of 1 and the alternative "driving at the right side" is attached a social index of 0 ; the social indices of the actions are reversed if the player believes the opponent drives on the right side of the road (in the U.S.). Through its dependence on the set of strategies, the convention is sensitive to the overall structure of a game, including the availability of strictly dominated strategies. In the doctor's example, suppose in addition to the effective drug, there are two equally ineffective physical treatments, $A$ and $B, A$ is even more expensive than $B$. In the absence of $A$, recommending $B$ is attached a social index of 0 ; but if $A$ is also an option for the doctor, then $B$ would be attached a positive social index since $A$ is now the 0 action. I allow for the possibility that one's entire set of pure actions is equally appropriate, which I refer to as the "trivial convention" case, and model it by giving all actions a social index of 199 Condition 2 extends the specification to mixed strategies by taking expectations of the corresponding social indices 10

I explore the idea that in principle, one would like to conform to conventions, but such effort is greatly affected by whether others conform to conventions too. Given common knowledge of the convention, $i$ 's belief of the social index of $j$ 's action is $\omega_{j}\left(b_{j} ; c_{i}\right)$, a function of $i$ 's first and second-order beliefs. Let $f_{i}\left(\sigma_{i}, b_{j}, c_{i}\right)=$ $\max \left\{0, \omega_{i}\left(\sigma_{i} ; b_{j}\right)-\omega_{j}\left(b_{j} ; c_{i}\right)\right\}$. This function represents $i$ 's belief about how much more his own action conforms to the convention comparing to his opponent's. I consider the following utility specification:

$$
u_{i}\left(\sigma_{i}, b_{j}, c_{i} ; \omega\right)=\pi_{i}\left(\sigma_{i}, b_{j}\right)+\theta_{i}\left[g_{i}\left(\omega_{i}\left(\sigma_{i} ; b_{j}\right)\right)+h_{i}\left(f_{i}\left(\sigma_{i}, b_{j}, c_{i}\right)\right)\right]
$$

\footnotetext{
${ }^{9}$ Under the trivial convention, the social implications of actions do not affect players' decisions.

${ }^{10}$ There are some conceptual subtleties when it comes to mixed strategies in this framework. For instance, one can imagine that believing the opponent is mixing 50/50 and assigning a 50/50 chance to the opponent playing either of two pure strategies would make a difference when making value judgement about the social implications of the opponent's action. The definition seems most appropriate if mixed strategy is interpreted as randomization over pure strategies.
} 
where $g_{i}, h_{i}:[0,1] \rightarrow \mathbb{R}$ are continuous, and satisfy:

1. Conformity: $g_{i}$ is increasing in $\omega_{i}$;

2. Interaction: $h_{i}$ is decreasing in $f_{i}$;

3. Total Concavity: $g_{i}+h_{i}$ is concave in $\omega_{i}$.

Equation (1) says players get utility from both material consequences and social implications of their actions, with the total payoff being a weighted sum of the two. The scalar $\theta_{i} \in[0, \infty)$ represents how salient the convention is to $i$ in the game. The larger $\theta_{i}$ is, the more $i$ takes social implications of actions into account when making decisions. A player with $\theta=0$ represents a classic agent who only cares about the material payoffs, while a player with $\theta \rightarrow \infty$ is an extremely sensitive person who only cares about the convention and ignores material consequences of actions 11

While $\theta_{i}$ captures the magnitude of social payoffs, the functions $g_{i}$ and $h_{i}$ capture the pattern of preferences for conventions. The function $g_{i}$ reflects the conformity effects of conventions: taking the appropriate action makes one happy. The function $h_{i}$ reflects the interaction effects of conventions: one prefers the opponent to conform to the conventions, and the less the opponent conforms to the conventions compared to oneself, the more disutility one receives from the interaction ${ }^{12}{ }^{13}$ Notice that since

\footnotetext{
${ }^{11}$ However, such a player may take the socially "wrong" action in equilibrium - see examples in Section 3.

${ }^{12}$ Reciprocity modeled in Rabin (1993) and concern withdrawal modeled in Charness and Rabin (2002) reflect the idea that one's belief about how kind the opponent is affects how kind one wants to be towards the opponent, which is similar to the interaction effects modeled here. Rabin (1993) assumes beliefs determine the sign and magnitude of payoffs derived from being kind to the opponent; Charness and Rabin (2002) let beliefs affect what actions are considered kind. The interaction affects in this model are closer to Rabin's in the sense that beliefs affect one's incentives to conform to the convention instead of the convention itself.

Intuitively, conventions, social norms, or ethical principles are by definition commonly-agreed behavioral codes. Their strength lies in the fact that they are respected by all members in society. The interaction effects capture the intuitive idea that one would like to conform to a behavioral code only if all other members conform to it as well. For example, while most people would treat even a stranger politely, they tend to be less polite with a rude acquaintance.

${ }^{13}$ The "objective social standard" in Charness and Rabin (2002) refers to the weight people put on social payoffs, the counterpart of $\theta_{i}$ in this model. Players punish those opponents whose actions seem to suggest a $\theta_{i}$ lower than some $\theta^{*}$, despite of the assumption of homogeneous preferences, by adjusting the rankings of actions to reflect a lack of concern for such opponents. For comparison, in this model, the convention itself is the social standard. Players "punish" the opponents in equilibrium, by not conforming to the convention themselves, if the opponents' equilibrium actions are ranked low. In some sense, players in this model are "consequentialists:" they don't care about how important social payoffs are in their opponents' decisions, instead, they only care about eventually how "correct" their actions are.
} 
$f_{i}$ is nonincreasing in $\omega_{j}\left(b_{j} ; c_{i}\right)$, for any $G$, the associated social game described by $g+h$ exhibits strategic complementarity in the social indices of equilibrium strategies ${ }^{14}$

For any two-person normal form game $G$, equation (1) defines the induced game with a convention $\omega$, denoted by $(G, \omega)$. Under equation (1), one's utility not only depends on material payoffs, but also depends on the social index of one's own action, which is belief-dependent, and one's belief about the social index of the opponent's action. Since the first and second-order beliefs enter the utility functions directly, the game is a psychological game. Adapting the psychological Nash equilibrium defined in GPS, I consider the following equilibrium concept:

Definition 2 A strategy profile $\sigma^{*}=\left(\sigma_{1}^{*}, \sigma_{2}^{*}\right) \in \Sigma$ is a social equilibrium in $(G, \omega)$ if, for $i, j=1,2$,

$$
\sigma_{i}^{*} \in \underset{\sigma_{i} \in \Sigma_{i}}{\arg \max } u_{i}\left(\sigma_{i}, \sigma_{j}^{*}, \sigma_{i}^{*} ; \omega\right)
$$

That is, a social equilibrium in $(G, \omega)$ is a Nash equilibrium that satisfies an additional consistency condition that all beliefs correspond to actual strategies. Since the social index is linear in probability, under total concavity, standard argument delivers the existence of social equilibrium:

Theorem 1 Social equilibrium exists for all $\theta_{1}, \theta_{2}$.

However, the set of social equilibria in $(G, \omega)$ can differ greatly from the set of Nash equilibria in $G$. Obviously, when $\theta_{i}=0, i=1,2$, social equilibrium reduces to Nash equilibrium. On the other hand, given the convention, not every Nash equilibrium may be approached by social equilibria.

\begin{tabular}{|c|c|c|}
\hline & $\mathrm{L}$ & $\mathrm{R}$ \\
\hline $\mathrm{U}$ & 0,0 & $1,-4$ \\
\hline $\mathrm{D}$ & 0,3 & 2,5 \\
\hline
\end{tabular}

Figure 1: Nash equilibrium and social equilibrium

For example, consider the game depicted in figure 1. This game has two pure strategy Nash equilibria: $(U, L)$ and $(D, R)$. Suppose the social indices are such that $\omega_{1}(D, L)=\omega_{2}(L, U)=1$, that is, it is appropriate for player 1 to play $D$ when player 2 plays $L$ and for player 2 to play $L$ when player 1 plays $U$. As long as $g_{1}(1)-g_{1}(0)$ and $\theta_{1}$ are positive, $(U, L)$ cannot be a social equilibrium. Intuitively, if a Nash equilibrium in $G$ involves material payoff ties for some player, then social implications of the relevant actions become pivotal in $(G, \omega)$.

In general, little can be said about which Nash equilibria are robust to the introduction of conventions, except in situations where all players have strict material

\footnotetext{
${ }^{14} \mathrm{~A}$ game has strategic complementarity if higher action of one player implies the other players gain more from taking higher actions as well.
} 
interests at stake: a strict Nash equilibrium is a social equilibrium as long as the salience parameters $\theta_{1}, \theta_{2}$ are sufficiently small, regardless of the convention involved.

It is perhaps of more interest to examine what happens when a convention is very salient to both players. Clearly, when $\theta_{1}, \theta_{2}$ are sufficiently large, players effectively play the associated social game described by $g+h$. By strategic complementarity, if there exists a (pure) strategy profile where both players' actions are social best responses to the opponent's action, then it is a social equilibrium regardless of the material consequences.

\section{Distributional Convention}

There are many social principles guiding different aspects of human life. In the rest of the paper, I focus attention on the principles most relevant to economic activities, namely efficiency and fairness, where efficiency principle applies to the total social surplus, and fairness principle applies to the allocation of payoffs between players, dubbed distributional convention 15

Let $\alpha_{e}(s)$ index the degree of efficiency in the outcome $\left(\pi_{1}(s), \pi_{2}(s)\right)$ and $\alpha_{f i}(s)$ index the degrees of $i$ 's fairness towards $j$ in this allocation. I define the social index to be the normalization of the weighted average: $\alpha_{i}(s)=t \alpha_{e}(s)+(1-t) \alpha_{f i}(s)$, where $t \in[0,1]$ is the weight society attaches to efficiency in evaluating the desirability of the outcomes.

The task is to define sensible measures for efficiency and fairness. Choosing an index for efficiency is less controversial. I use the following measure:

$$
\alpha_{e}(s)=\left\{\begin{array}{cc}
\frac{\sum_{i} \pi_{i}(s)-\min _{s \in S} \sum_{i} \pi_{i}(s)}{\max _{s \in S} \sum_{i} \pi_{i}(s)-\min _{s \in S} \sum_{i} \pi_{i}(s)} & \text { if } \max _{s \in S} \sum_{i} \pi_{i}(s) \neq \min _{s \in S} \sum_{i} \pi_{i}(s) \\
0 & \text { if } \max _{s \in S} \sum_{i} \pi_{i}(s)=\min _{s \in S} \sum_{i} \pi_{i}(s)
\end{array}\right.
$$

Choosing a measure for fairness, however, is more complicated. In two-person games, equity seems to be a fairly good proxy. But equity is a symmetric measure and would indicate that a person who chooses (self, other) allocations of $(1,3)$ over $(2,2)$ violates fairness, and violates it as much as choosing $(3,1)$. This contradicts the usual perception of being fair. It seems "fairness," in its common usage, incorporates not only the notion of equity, but also a sense of being "generous" or "humble" towards others. In the above example, one can argue that a person choosing $(1,3)$ over $(2,2)$

\footnotetext{
${ }^{15}$ Distributional convention reflects social criteria about the desirability of different payoff allocations. The specification is similar to distributional preferences as discussed in Charness and Rabin (2002) and in other distributional models. The difference is that, instead of being interpreted as individual preferences, here it is interpreted as a social ranking of outcomes. Also see footnote 19 for the connection with the literature.
} 
is unequal to himself, but is generous to the opponent, which is considered "fair." Let

$$
\alpha_{q}(s)=\left\{\begin{array}{cl}
1 & \text { if } \pi_{i}=\pi_{j}=0 \\
1-\frac{\left|\pi_{i}-\pi_{j}\right|}{\left|\pi_{i}\right|+\left|\pi_{j}\right|} & \text { otherwise }
\end{array}\right.
$$

index the degree of equity of outcomes. It decreases in the difference between two payoffs, equals 1 whenever two players receive identical payoffs, and equals 0 whenever one player receives 0 or negative payoffs while the other player receives positive payoffs $\sqrt{16}$ Let

$$
\alpha_{g i}(s)=\left\{\begin{array}{cl}
0 & \text { if } \pi_{i}=\pi_{j}=0 \\
\max \left\{\frac{\pi_{j}-\pi_{i}}{\left|\pi_{i}\right|+\left|\pi_{j}\right|}, 0\right\} & \text { otherwise }
\end{array}\right.
$$

index $i$ 's generosity considerations. The index is zero whenever one grabs more than the opponent, and increases in the amount the opponent leads. With this adjustment, choosing an unequal allocation to one's own disadvantage is not unfair. I define fairness as a weighted average of equity and generosity considerations: $\alpha_{f i}(s ; r)=$ $r \alpha_{q}(s)+(1-r) \alpha_{g i}(s)$ where $r \in[0,1]$. For $r=\frac{1}{2}$, it is fair whenever one gives the opponent weakly more than one's own payoffs. The fairness index $\alpha_{f i}$ increases in $\pi_{j}$ for all $\pi_{j}<\pi_{i}$ and reaches maximum for all $\pi_{j} \geq \pi_{i}$. For $r<\frac{1}{2}$, the fairness criterion values generosity: the more one gives, the fairer one is - the fairness index increases in $\pi_{j}-\pi_{i}$. Finally, for $r>\frac{1}{2}$, letting others lead is not encouraged, and the fairness index obtains the maximum at $\pi_{j}=\pi_{i}$. In this case, the fairness index reflects inequality aversion. ${ }^{17}{ }^{18}$

The social index for $i$ taking pure action $s_{i}$, believing $j$ plays pure action $b_{j}$ is:

$$
\omega_{i}\left(s_{i}, b_{j}\right)=\left\{\begin{array}{cl}
1 & \text { if } \max _{s_{i} \in S_{i}} \alpha_{i}\left(s_{i}, b_{j}\right)=\min _{s_{i} \in S_{i}} \alpha_{i}\left(s_{i}, b_{j}\right) \\
\frac{\alpha_{i}\left(s_{i}, b_{j}\right)-\min _{s_{i} \in S_{i}} \alpha_{i}\left(s_{i}, b_{j}\right)}{\max _{s_{i} \in S_{i}} \alpha_{i}\left(s_{i}, b_{j}\right)-\min _{s_{i} \in S_{i}} \alpha_{i}\left(s_{i}, b_{j}\right)} & \text { otherwise }
\end{array}\right.
$$

where $\alpha_{i}\left(s_{i}, b_{j}\right)=t \alpha_{e}\left(s_{i}, b_{j}\right)+(1-t)\left[r \alpha_{q}\left(s_{i}, b_{j}\right)+(1-r) \alpha_{g i}\left(s_{i}, b_{j}\right)\right]$ and $\alpha_{e}, \alpha_{q}, \alpha_{g i}$ are as defined above 19

\footnotetext{
${ }^{16}$ The index seems reasonable for games with nonnegative payoffs. It works less satisfactorily when there are negative payoffs involved.

${ }^{17}$ As long as $r>0$, letting others lead is ranked higher than leading oneself by the same amount.

${ }^{18}$ The generosity consideration introduces an intrinsic asymmetry of social implications of players' actions in any action profile: $\pi_{j}(s)>\pi_{i}(s)$ implies $\alpha_{f i}(s)>\alpha_{f j}(s)$, and this difference decreases in $r$.

${ }^{19}$ The distributional convention is similar to the distributional preferences studied in Charness and Rabin (2002), in particular, the combination of efficiency and fairness bears similarity to the combination of efficiency and maximin in their model. For different values of $t$ and $r$, the distributional convention replicates some other criteria suggested in the literature, too. For example, for $t=1$, the distributional convention reflects the social welfare criterion; for $t=0, r>\frac{1}{2}$, it reflects inequality aversion.
} 
The presence of strategic complementarity in preferences for distributional convention gives rise to behavior reflecting social preferences in social equilibrium. Intuitively, there are two trade-offs in games with conventions: the trade-off between material payoffs and social payoffs; and the trade-off between utility derived from conformity effects and the disutilities derived from interaction effects. If conformity effects dominate, one would prefer to sacrifice the material payoffs in order to conform to distributional convention in social equilibrium, resulting in altruistic type behavior; while if interaction effects dominate, one would prefer to take a low-ranking action at the cost of material payoffs, which then is interpreted intuitively as retaliatory behavior ${ }^{20}$ It is easy to see that given the distributional convention, the "most" efficient and fair outcome, had it existed, can be supported in social equilibrium as long as the convention is sufficiently salient for both players. ${ }^{21}$

I apply the model to two classes of games that have attracted much attention in both experimental and theoretical work: symmetric $2 \times 2$ games, including the prisoner's dilemma game, the coordination game and the chicken game; and publicgood games, including the summation game and the best-shot game. I restrict my attention to pure strategy social equilibrium throughout.

\subsection{Symmetric $2 \times 2$ Games}

Figure 2 depicts the symmetric $2 \times 2$ game, where "C" stands for cooperate and "D" stands for defect. Let the payoff entries represent the monetary payoffs players receive, and that $a, b, c, d>0, a>b, c>d$. Three particularly important games in this class include the prisoner's dilemma game $(b<d<c<a)$, the pure coordination game $(b<d<c, a<c)$ and the chicken game $(c<a, d<b, d<c)$. I am interested in whether and under what conditions $(C, C)$ is a (unique) social equilibrium in the induced game with distributional convention.

\begin{tabular}{|c|c|c|}
\hline & $\mathrm{C}$ & $\mathrm{D}$ \\
\hline $\mathrm{C}$ & $c, c$ & $b, a$ \\
\hline $\mathrm{D}$ & $a, b$ & $d, d$ \\
\hline
\end{tabular}

Figure 2: symmetric $2 \times 2$ games

The strategy profile $(C, C)$ is a Nash equilibrium in the coordination game, but not unique; it is not a Nash equilibrium in the prisoner's dilemma game and the chicken game. These predictions have been long regarded as inaccurate and counterintuitive, especially in the prisoner's dilemma game, where the unique equilibrium

\footnotetext{
${ }^{20}$ Notice that the latter observation links the relative strength of conformity effects and the interaction effects in one's preference for conventions with retaliatory behavior in social equilibrium in games with distributional convention.

${ }^{21}$ Recall footnote 18 For a strategy profile $s$ such that $\omega_{i}\left(s_{i} ; s_{j}\right)=1$ for $i=1,2$ to exist under distributional convention, a necessary condition is that $r$ cannot be too small.
} 
outcome is Pareto dominated by the cooperation outcome. In fact, in laboratory experiments of one-shot or finitely repeated prisoner's dilemma games, cooperation outcomes are frequently observed, while the ratio of cooperation outcomes typically depends on fine details of the payoff structure of the game (Cooper, DeJong, Forsythe and Ross 1996, Dawes and Thaler 1988, Georee and Holt 2001).

An intuitive explanation is that the game is in fact not prisoner's dilemma from the subjects' perspective due to their considerations of things other than the material payoff entries. ${ }^{22}$ The challenge is to "expand" the game appropriately to account for the dependence of the equilibrium in the "real" game on fine details of the original game.

As a benchmark, consider the pure coordination game with distributional convention. It is easy to see that Nash equilibria in this game are robust to the introduction of distributional convention for all values of $t, r$ and $\theta_{1}, \theta_{2}$, because the distributional convention is perfectly aligned with material interests in the game: for all $t, r, i=1,2, \omega_{i}(C, C)=\omega_{i}(D, D)=1$. The interesting thing is now $(C, D)$ and $(D, C)$ can be social equilibria, too.

For concreteness, consider the following utility function:

$$
u_{i}\left(s_{i}, b_{j}, c_{i} ; \omega\right)=\pi_{i}\left(s_{i}, b_{j}\right)+\theta_{i}\left[-\left(1-\omega_{i}\right)^{2}-4 \max \left\{\omega_{i}\left(s_{i}, b_{j}\right)-\omega_{j}\left(b_{j}, c_{i}\right)\right\}\right]
$$

The quadratic loss function $-\left(1-\omega_{i}\right)^{2}$ captures the conformity effects, and the linear loss function $-4 \max \left\{\omega_{i}\left(s_{i}, b_{j}\right)-\omega_{j}\left(b_{j}, c_{i}\right)\right\}$ captures the interaction effects. Let $c=6, a=b=0, d=3$. Then $(D, C)$ is a social equilibrium as long as $0-\theta_{1} \geq$ $6-4 \theta_{1}, 0-\theta_{2} \geq 3-4 \theta_{2}$, or $\theta_{1} \geq 2$ and $\theta_{2} \geq 1$. Intuitively, one could supply a retaliation story for this equilibrium: 1 believes 2 believes 1 plays $D$, which means 2 is disregarding the convention, perhaps in order to hurt 1 . If the convention is sufficiently salient to $1\left(\theta_{1} \geq 2\right)$, even though playing $C$ is materially beneficial, the disutility from conforming to the convention given 2 does not is so much that 1 would rather play $D$. Similarly for 2 . Since 2's material stake is smaller than 1 , the required salience for 2 is also lower. Moreover, the equilibrium depends on fine material payoff details in the game: as the material stakes $a-c$ or $d-b$ increases, it is harder and harder to sustain $(D, C)$ as a social equilibrium: the required salience values of $\theta_{1}$ and $\theta_{2}$ increase in $a-c$ and $d-b$ respectively. Now consider the prisoner's dilemma game and the chicken game with distributional convention. Let $c=6, a=8, b=4, d_{1}=5$ (the prisoner's dilemma) and $d_{2}=-5$ (the chicken game). Obviously, for all $t, r$, $\omega_{i}(C, C)=1, i=1,2$ in both games. That is, it is conventional to cooperate if the opponent cooperates. It follows that in both games, $(C, C)$ is social equilibrium provided $\theta_{i} \geq 2, i=1,2$. In this social equilibrium, players appear to be altruistic towards each other. Moreover, the higher $a$ is, the harder it is to sustain $(C, C)$ as a social equilibrium: first of all, higher $a$ increases the material temptation $a-c$; second, for sufficiently high $a$, the outcome $(a, b)$ could be so efficient that the distributional

\footnotetext{
${ }^{22}$ For an inspiring discussion about fundamental modeling issues in game theory, see Weibull(2004).
} 
convention would rank $D$ above $C$, in which case, for all $\theta_{1}, \theta_{2},(C, C)$ cannot be a social equilibrium 23

Whether there is other social equilibrium depends on how the distributional convention ranks $C$ and $D$ when the opponent plays $D$. Suppose efficiency matters in the distributional convention $(t>0)$ and the generosity consideration is not over-emphasized in fairness criterion ( $r \geq \frac{1}{2}$ is a sufficient condition). Then it is conventional to cooperate even if the opponent defects: $\omega_{i}(C ; D)=1, i=1,2$. Under such convention, in the prisoner's dilemma game, $(D, D)$ is social equilibrium for all values of $\theta_{1}, \theta_{2}$. The reason is similar to that given for $(D, C)$ being the social equilibrium in the pure coordination game: both players believe the opponent does not conform to the convention, and hence given the interaction effects in social payoffs, it is beneficial to defect not only in terms of material payoffs, but also in terms of social payoffs. On the other hand, $(C, D)$ or $(D, C)$ can never be social equilibrium. This is because this strategy profile has a social index profile of $(0,1)$, where 1 does not conform to the convention but 2 does, given each other's action. But then 2 would like to deviate: given the interaction effects, 2 prefers not to conform to the convention when 1 does not; besides, it is also materially beneficial to do so.

Similarly, one can show that, for different combinations of values of $\theta_{1}, \theta_{2}$, the sets of pure strategy social equilibria in the chicken game are as follows:

\begin{tabular}{|c|c|c|c|c|}
\hline & $\theta_{2} \in[0,1]$ & {$[1,2]$} & {$[2,3]$} & {$[3, \infty)$} \\
\hline$\theta_{1} \in[0,1]$ & $\{(C, D),(D, C)\}$ & $\{(C, D)\}$ & $\emptyset$ & $\emptyset$ \\
\hline$[1,2]$ & $\{(D, C)\}$ & $\emptyset$ & $\emptyset$ & $\emptyset$ \\
\hline$[2,3]$ & $\emptyset$ & $\emptyset$ & $\{(C, C)\}$ & $\{(C, C)\}$ \\
\hline$[3, \infty)$ & $\emptyset$ & $\emptyset$ & $\{(C, C)\}$ & $\{(C, C),(D, D)\}$ \\
\hline
\end{tabular}

It is worth noting that when $\left(\theta_{1}, \theta_{2}\right) \in(2, \infty)^{2} \backslash[3, \infty)^{2},(C, C)$ is the unique social equilibrium. To see why this is the case, notice that, in this game, "punishing" an opponent who does not conform to the convention is more costly than "rewarding" an opponent who conforms to the convention $(d-b>a-c)$. This creates room for $C$ to be the dominant strategy for a suitably convention-conscientious player, who values social payoffs enough to prefer to sacrifice $a-c$ in order to "reward" a "kind" opponent, yet does not value it that much to be willing to give up $d-b$ to "punish" an "unworthy" opponent.

On the other hand, retaliatory behavior in equilibrium depends on the relative strength of conformity effects and interaction effects in players' social payoffs. For example, consider :

$$
u_{i}\left(s_{i}, b_{j}, c_{i} ; \omega\right)=\pi_{i}\left(s_{i}, b_{j}\right)+\theta_{i}\left[-2\left(1-\omega_{i}\right)^{2}-\max \left\{\omega_{i}\left(s_{i}, b_{j}\right)-\omega_{j}\left(b_{j}, c_{i}\right)\right\}\right]
$$

\footnotetext{
${ }^{23}$ When $a+b>2 c$, there is a trade-off between efficiency and fairness. Suppose $c=10, a=$ $1000, b=9$. Is $(10,10)$ necessarily a better outcome than $(1000,9)$ ? It depends on how society evaluates efficiency versus equity, namely, the value of $\frac{t}{(1-t) r}$. For details, see the proofs for section 3.1 in the appendix.
} 
With this utility function, $i$ would prefer to conform to the convention even if the opponent does not conform to it. It is easy to see that now in the pure coordination game, $(C, D)$ or $(D, C)$ cannot be social equilibria. For all values of $\theta_{1}, \theta_{2}$, the set of social equilibria in this game is $\{(C, C),(D, D)\}$. In the prisoner's dilemma game, suppose it is always conventional to cooperate, regardless of the opponent's action, then $(D, D)$ is social equilibrium only when the convention is not very salient for both players $\left(\theta_{i} \leq 1, i=1,2\right) ;(C, D)$ could be social equilibrium if the convention is salient for 1 but not for $2\left(\theta_{1} \geq 1, \theta_{2} \leq 1\right)$, similarly for $(D, C)$. In this case, it is actually possible to achieve $(C, C)$ as the unique social equilibrium even in the prisoner's dilemma game $\left(\theta_{i}>1, i=1,2\right)$. In the chicken game, again suppose the convention ranks $C$ above $D$ regardless of the opponent's action, then $(D, D)$ is never social equilibrium, $(C, D)$ is social equilibrium provided the convention is not very salient for $2\left(\theta_{2} \leq 1\right)$, similarly for $(D, C)$. In this case $(C, C)$ is the unique social equilibrium whenever the convention is sufficiently salient for both players $\left(\theta_{i}>1, i=1,2\right)$.

The above analysis illustrates how equilibrium behavior depends on fine details of material payoff structure in the game, the prevailing convention $(t, r)$, the salience of conventions for each player $\left(\theta_{1}, \theta_{2}\right)$, and the relative strength of conformity effects and interaction effects in the social payoffs $\left(g_{i}, h_{i}\right)$. The predictions are much richer and more realistic than that in the traditional analysis, yet the complex behavioral patterns can be organized concisely as the following results.

Theorem 2 In the symmetric $2 \times 2$ game with distributional convention, the strategy profile $(C, C)$ is a social equilibrium if and only if one of the following conditions holds:

1. $2 c \geq a+b$ and $\theta_{i} \geq \frac{a-c}{g_{i}(1)-g_{i}(0)}$ for $i=1,2$;

2. $2 c<a+b, \frac{(1-t) r}{t}>\frac{(a+b-2 c)(a+b)}{(a+b-2 d)(a-b)}$ and $\theta_{i} \geq \frac{a-c}{g_{i}(1)-g_{i}(0)}$ for $i=1,2$;

3. $2 c<a+b, \frac{(1-t) r}{t}<\frac{(a+b-2 c)(a+b)}{(a+b-2 d)(a-b)}$; and $g_{i}(1)-g_{i}(0)<h_{i}(0)-h_{i}(1), \theta_{i} \geq$ $\frac{a-c}{g_{i}(0)+h_{i}(0)-\left(g_{i}(1)+h_{i}(1)\right)}$ for $i=1,2$.

This result gives the necessary and sufficient conditions for coordination outcome to be a social equilibrium: either it is conventional to cooperate when the opponent cooperates, and such convention is sufficiently salient for both players (conditions 1 and 2); or it is conventional to defect when the opponent cooperates, and the interaction effects dominate the conformity effects in players' social payoffs (condition $3)$.

The second result summarizes the situations where $(C, C)$ can be obtained as the unique social equilibrium.

Theorem 3 In the symmetric $2 \times 2$ normal-form game with distributional convention, suppose $d>b$. Then $(C, C)$ is the unique social equilibrium if and only if, 
1a. $\frac{(1-t) r}{t}>\frac{(a+b-2 c)(a+b)}{(a+b-2 d)(a-b)}$

$\frac{(1-t)(2 r-1)}{t}<\frac{|a+b-2 d|(a+b)}{(2 c-\min \{a+b, 2 d\})(a-b)}$ if $2 c \geq a+b$ and $\frac{(1-t)(2 r-1)}{t}<\frac{(a+b)}{(a-b)}$ otherwise;

1b. $g_{i}(1)-g_{i}(0)>h_{i}(0)-h_{i}(1)$ for $i=1,2$;

1c. $\theta_{i}>\max \left\{\frac{a-c}{g_{i}(1)-g_{i}(0)}, \frac{b-d}{g_{i}(0)+h_{i}(0)-\left(g_{i}(1)+h_{i}(1)\right)}\right\}$ for $i=1,2$.

Suppose $d<b$. Then $(C, C)$ is the unique social equilibrium provided,

2a. $\frac{(1-t) r}{t}>\frac{(a+b-2 c)(a+b)}{(a+b-2 d)(a-b)}$;

$\frac{(1-t)(2 r-1)}{t}<\frac{|a+b-2 d|(a+b)}{(2 c-2 d)(a-b)}$ if $2 c \geq a+b ;$ and $\frac{(1-t)(2 r-1)}{t}<\frac{(a+b)}{(a-b)}$ otherwise;

2b. for $i=1,2, \theta_{i} \in\left(\frac{a-c}{g_{i}(1)-g_{i}(0)}, \infty\right)$ if $g_{i}(1)-g_{i}(0)>h_{i}(0)-h_{i}(1)$; and $\theta_{i} \in$ $\left(\frac{a-c}{g_{i}(1)-g_{i}(0)}, \frac{b-d}{g_{i}(0)+h_{i}(0)-\left(g_{i}(1)+h_{i}(1)\right)}\right)$ otherwise;

or,

3a. $\frac{(1-t) r}{t}>\frac{(a+b-2 c)(a+b)}{(a+b-2 d)(a-b)}$;

$\frac{(1-t)(2 r-1)}{t}>\frac{|a+b-2 d|(a+b)}{(2 c-2 d)(a-b)}$ if $2 c \geq a+b ;$ and $\frac{(1-t)(2 r-1)}{t}>\frac{(a+b)}{(a-b)}$ otherwise;

3b. $g_{i}(1)-g_{i}(0)>h_{i}(0)-h_{i}(1)$ for $i=1,2$;

3c. $\theta_{i} \in\left(\frac{a-c}{\left|g_{i}(0)+h_{i}(0)-\left(g_{i}(1)+h_{i}(1)\right)\right|}, \frac{b-d}{g_{i}(1)-g_{i}(0)}\right)$ for $i=1,2$.

The first set of conditions characterize the necessary and sufficient conditions for $(C, C)$ to be a unique social equilibrium in symmetric $2 \times 2$ games with distributional convention that satisfy the condition that it is materially beneficial to play $D$ when the opponent plays $D{ }^{24}$ the distributional convention ranks $C$ above $D$ regardless of the opponent's action (condition 1a); the conformity effects dominate the interaction effects in players' social payoffs (condition 1b); and the convention is sufficiently salient for both players (condition 1c). The second and third sets of conditions give the sufficient conditions for $(C, C)$ to be the unique social equilibrium depending on the prevailing distributional conventions in games where the material payoff structure satisfies $d<b \sqrt{25}$ if it is conventional to play $C$ regardless of the opponent's action (condition 2a), then for $(C, C)$ to be the unique social equilibrium, such conventions need to be salient but not overwhelmingly so (condition $2 \mathrm{~b}$ ); if it is conventional to play $C$ when the opponent plays $C$ but to play $D$ when the opponent plays $D$ (condition 3a), then not only the convention needs to be suitably salient (condition $3 \mathrm{~b}$ ), but also the conformity effects need to dominate the interaction effects in social payoffs (condition 3c).

\footnotetext{
${ }^{24}$ Note this includes the pure coordination games and the prisoner's dilemma games.

${ }^{25}$ This includes the chicken game.
} 
Given the large amount of experimental evidence of retaliatory behavior, I am particularly interested in equilibrium behavior of players whose preferences for conventions are such that the interaction effects dominate the conformity effects.

Corollary 4 In the symmetric $2 \times 2$ game with distributional convention, suppose $h_{i}(0)-h_{i}(1)>g_{i}(1)-g_{i}(0)$ for $i=1,2$. Then:

1. $(C, C)$ is a social equilibrium if and only if: (1) $2 c \geq a+b$ or $\frac{(1-t) r}{t}>\frac{(a+b-2 c)(a+b)}{(a+b-2 d)(a-b)}$ for $2 c<a+b$; and (2) $\theta_{i} \geq a-c, i=1,2$;

2. $(C, C)$ is the unique equilibrium only if: (1) $d-b>a-c$; (2) $\frac{(1-t) r}{t}>$ $\frac{(a+b-2 c)(a+b)}{(a+b-2 d)(a-b)}$; (3) $\frac{(1-t)(2 r-1)}{t}<\frac{|a+b-2 d|(a+b)}{(2 c-2 d)(a-b)}$ if $2 c \geq a+b$; and $\frac{(1-t)(2 r-1)}{t}<\frac{(a+b)}{(a-b)}$ otherwise.

The result states that, when interaction effects dominate conformity effects in both players' social payoffs, $(1)$ the necessary and sufficient conditions for $(C, C)$ to be a social equilibrium is that, it is conventional to cooperate when the opponent cooperates, and such convention is sufficiently salient for both players; (2) only in the chicken game it is possible to achieve $(C, C)$ as a unique social equilibrium, for which a necessary condition is that it is conventional to cooperate regardless of one's beliefs about the opponent's action.

\subsection{The Public-good Games}

Two players simultaneously decide how much to contribute to the public good. If player $i$ contributes $c_{i} \leq m$ to the public good $C$, his material payoff is $\pi_{i}\left(c_{i} ; c_{j}\right)=m-$ $c_{i}+\left(p_{i}+1\right) \ln C\left(c_{i} ; c_{j}\right)$. I examine two variants of the game: the summation game where $C=c_{1}+c_{2}+1$; and the best-shot game where $C=\max \left\{c_{1}, c_{2}\right\}+1 \cdot{ }^{26}{ }^{27}$ Let $p_{1}>>p_{2}$ so that $\left(p_{1}, 0\right)$ is the only Nash equilibrium in both games. The public-good games are extensively explored in the literature, for example, Harrison and Hirshleifer (1989), Prasnikar and Roth (1992), Andreoni, Brown and Vesterlund (2002), among others. It is found that typically subjects do not play the Nash equilibrium in experiments of the summation game, while they do in the best-shot game.

Intuitively, this is because in the two games, 2's free-riding behavior has different social implications. Given 1 contributes a positive amount $c_{1}>0$, the efficiency index of 2's action increases in $c_{2}$ in the interval $\left[0, p_{1}+p_{2}+1-c_{1}\right]$ in the summation game, while decreases in $c_{2}$ in the best-shot game. In other words, according to the efficiency criterion, when 1 contributes much to the public good, for 2 , free-riding is

\footnotetext{
${ }^{26}$ In the literature, the best-shot game typically refers to a dynamic public-good game where players move sequentially and the public good is supplied at the maximum of all private contributions. I deal with the normal-form version to avoid complications arising from sequential moves.

${ }^{27}$ The typical utility function used in this class of games is $\pi_{i}\left(c_{i} ; c_{j}\right)=m-c_{i}+p_{i} \ln C$ where $C=c_{1}+c_{2}$ or $C=\max \left\{c_{1}, c_{2}\right\}$. The modification is because I need a bounded function.
} 
the right thing to do in the best-shot game, but not in the summation game, in fact, it is the least appropriate action in that situation.

The distributional convention captures this subtlety. For simplicity, let $t=1$, i.e. efficiency is the only underlying principle of distributional convention. Under such convention, the right thing to do is to contribute as much as it takes to reach the social optimal level of the public good, i.e. $p_{1}+p_{2}+1$. Thus, there exists some $c_{1}^{*} \in\left(0, p_{1}\right)$ such that for all $c_{1} \in\left[c_{1}^{*}, p_{1}+p_{2}+1\right], \omega_{1}^{S}\left(c_{1} ; 0\right)>\omega_{2}^{S}\left(c_{1} ; 0\right)=0$ while $\omega_{1}^{B}\left(c_{1} ; 0\right)<\omega_{2}^{B}\left(c_{1} ; 0\right)=1$, where $\omega_{i}^{S}, \omega_{i}^{B}$ denote the social index of $i$ 's action in the summation game and the best-shot game respectively. Since the social index of 1's contribution increases monotonically in the interval $\left[0, p_{1}+p_{2}+1\right]$ conditional on $c_{2}=0$, and 1's material payoff monotonically increases in the interval $\left[0, p_{1}\right]$, it is the interaction effects that could potentially upset such a social equilibrium. But for $c_{1}$ that falls in the above range, the interaction effects are only present in the summation game. Consequently in the best-shot game, a much wider range of $\theta_{1}, \theta_{2}$ are susceptible to a social equilibrium in which 2 free rides.

For a numerical example, let $t=1, p_{1}=9, p_{2}=3, m=20$ and consider the utility function 3. The equilibrium characterizations turns out to fit the experimental results very well (for example, as in Experiment 3 from the introduction (Andreoni, Brown and Vesterlund 2002)). I summarize the findings below.

First, in the best-shot game, for all $\theta_{1}, \theta_{2},(9,0)$ is a social equilibrium, while $\left(c_{1}, 0\right)$ where $4.8 \leq c_{1}<9$ is never a social equilibrium. In other words, if the prevailing convention attaches high weight to social efficiency, then it is always a social equilibrium for 2 to free ride and 1 to contribute the entire public good; moreover, in every social equilibrium 2 free rides, 1 would never contribute less than $\frac{1}{2}$ of his willingness-to-pay. On the other hand, 2 prefers to free ride as long as he believes 1 contributes at least 3 .

To the contrary, in the summation game, only for $\theta_{1}=\theta_{2}=0,(9,0)$ is a social equilibrium. In fact, if the convention is salient for $1\left(\theta_{1} \geq 6\right)$, he never contributes more than a negligible amount $\left(c_{1}<.89\right)$ in any social equilibrium in which 2 free rides. On the other hand, 2 typically does not want to free ride if he believes 1 contributes a positive amount, even if 1's contribution already exceeds 2's own marginal-willingness-to-pay. For example, given that 1 contributes 3,2 prefers to free ride only if his salience is extremely low $\left(\theta_{2} \leq 0.23\right)$. Intuitively, in these social equilibria, 2's social index is 0 while 1 's is positive, and hence 1 is subject to the interaction effects while 2 is subject to the conformity effects. It is possible to have such outcome as a social equilibrium only if neither player puts much weight on social payoffs, i.e. has low salience.

In the set of social equilibria in which 2 free rides $\left(c_{2}^{*}=0\right), 1$ 's equilibrium contribution as a function of the salience parameter $c_{1}^{*}\left(\theta_{1}\right)$ has very different properties in the two games. In the best-shot game, $c_{1}^{*}$ increases in $\theta_{1}$; while in the summation game, it decreases in $\theta_{1}$. To see this, notice that given 2 free rides, the sign of the social payoffs for 1 are different in the two games: it is negative in the summation 
game due to the interaction effects, while it is positive in the best-shot game due to the conformity effects. In particular, in the summation game, 1 contributes less than his marginal willingness-to-pay, while in the best-shot game, 1 contributes at least his marginal willingness-to-pay.

Finally, for $\theta_{1} \geq 6.7, \theta_{2} \geq 1.97,(0,0)$ is a social equilibrium in both games. In this equilibrium, both players' actions have a social index of 0 . As discussed before, a necessary condition for such a social equilibrium is that the interaction effects dominate the conformity effects in both players' social payoffs.

\section{Concluding remarks}

I propose a simple model to account for social preferences. The model takes "conventions" as given and hypothesizes that people prefer to conform to conventions, and prefer that the opponent conforms to conventions as well. Formalizing distributional convention as a belief-dependent ranking over the whole strategy space according to some combination of efficiency and fairness principles, I show that equilibrium behavior in games incorporating distributional convention reflects social preferences. For concrete examples, I show that the model makes sharp predictions in symmetric $2 \times 2$ games and public-good games that are consistent with experimental evidence.

The simplicity and parsimony of the model make it particularly appealing empirically. The separation of heterogeneous salience of convention and the convention itself, and the fact that the convention parameters are obviously experimentally manipulatable make it easy to empirically test the model. For example, in symmetric $2 \times 2$ games, holding everything else constant, the model predicts that equilibrium outcome varies with the material payoff details, for example, $a-c$; in particular, the model predicts distinctly different behavior when the material payoff structure is that of the chicken game. In the public-good game, the model predicts different sets of social equilibria in the two games for fixed salience parameters $\theta_{1}, \theta_{2}$.

Conceptually, the model also differs from the main body of the social preferences literature in that in a sense, inter-dependent utilities are not the primitives of the model. In this model, the presence and nature of inter-dependent utilities only reflect the presence and nature of exogenous conventions. In a social equilibrium, by taking into account the actions' social implications, which are evaluated according to some convention that depends on overall payoff allocations, players act as if they care about each other. The nature and pattern of "social preferences" reflect the nature and pattern of the prevailing distributional convention in the game. By manipulating the distributional convention in a game, one could change the pattern and/or degree of such interdependence. ${ }^{28}$

The model embraces an "instrumental view" of conventions in one-shot games that is reminiscent of the repeated games or evolutionary arguments for social prefer-

\footnotetext{
${ }^{28}$ For example, by manipulating the framing of the game.
} 
ences. One can view a convention as a coordination device: it suggests an appropriate action (or actions) to each player in each and every context; given the strategic complementarity of the social payoffs, for sufficiently convention-conscientious players, a fixed point of the "social best response" correspondence is a social equilibrium, achieving the outcome the convention targets. Furthermore, the concept of conventions is reminiscent of notions such as social norms, ethics, morals, etc. The model connects social preferences to questions such as, what social norms are sustainable in the long run? What determines the emergence and fading of particular moral principles? This suggests a thorough understanding of social preferences be based on a theory of evolution of social norms.

There are a number of interesting extensions one can study. Multi-person games and dynamic games are the most obvious ones. How do people respond to an environment where multiple opponents take actions with different social implications? How do people draw inferences when such inferences affect their utilities directly in dynamic games? How to evaluate the social implications of an action in these complex environments? These are open questions that invite both experimental and theoretical examinations. The current model provides a framework that highlights the additional questions that need to be answered and sheds light on possible experimental design.

In real life, people do not always share the same convention. For example, people from different cultural backgrounds may respect different ethical principles or attach different weights to even the same set of ethical principles, hence evaluate an action's social implications differently. One could imagine, bargaining impasse could result from each bargainer evaluating the social implications of actions using the convention most favorable to his own material benefits. Such issues could be dealt with in a tractable way using the framework presented in this paper, for example, by introducing payoff uncertainties into games with multiple conventions.

\section{Appendix}

\subsection{Proofs for results in the symmetric $2 \times 2$ game.}

These results can be obtained by examining the conditions for each of the four pure strategy profiles to be a social equilibrium case by case.

\section{Preliminary observations:}

Suppose the opponent plays $C$. First observe that $2 c \geq a+b$ is a sufficient condition for $\omega_{i}(C ; C)=1$ : given $j$ cooperates, defection would result in a less efficient and less equal outcome, where $i$ is grabbing more for himself. When $2 c>a+b$, the ranking is determined by the trade-off between efficiency and fairness. Cooperation 
is socially desirable if:

$$
t \frac{2 c-2 d}{a+b-2 d}+(1-t) r \geq t+(1-t) r\left(1-\frac{a-b}{a+b}\right)
$$

that is:

$$
\frac{(1-t) r}{t} \geq \frac{(a+b)(a+b-2 c)}{(a-b)(a+b-2 d)}
$$

This inequality is easier to satisfy when $t$ is small (society cares little about efficiency), or $a+b$ is close to $2 c$ (total social surplus does not increase much when a player unilaterally deviates to defect). To summarize, for $i=1,2$,

$$
\omega_{i}(C ; C)= \begin{cases}0 & \text { if } 2 c<a+b \text { and } \frac{(1-t) r}{t}<\frac{(a+b)(a+b-2 c)}{(a-b)(a+b-2 d)} \\ 1 & \text { otherwise }\end{cases}
$$

Suppose the opponent plays $D$. Straightforward computations yield:

$$
\omega_{i}(C ; D)= \begin{cases}0 & \text { if } 2 c<a+b \text { and } \frac{(1-t)(2 r-1)}{t}>\frac{(a+b)}{(a-b)} \\ 0 & \text { if } 2 c \geq a+b \text { and } \frac{(1-t)(2 r-1)}{t}>\frac{(a+b)|a+b-2 d|}{(a-b)(2 c-\min \{a+b, 2 d\})} \\ 1 & \text { otherwise }\end{cases}
$$

Observe that when $a+b>2 c$, since $2 r-1 \leq r$ and $\frac{a+b-2 c}{a+b-2 d}<1$, it follows $\omega_{i}(D ; C)=1 \Rightarrow \omega_{i}(C ; D)=1$ and $\omega_{i}(D ; D)=1 \Rightarrow \omega_{i}(C ; C)=1$.

For $(C, C)$ to be a social equilibrium: the relevant distributional convention is the ranking of $C$ and $D$ given the opponent plays $C$.

1. Suppose $2 c \geq a+b$, then $\omega_{i}(C ; C)=1$ and $\omega_{i}(D ; C)=0$. The equilibrium condition is:

$$
\begin{aligned}
c+\theta_{i}\left(g_{i}(1)+h_{i}(0)\right) & \geq a+\theta_{i}\left(g_{i}(0)+h_{i}(0)\right. \\
\theta_{i} & \geq \frac{a-c}{g_{i}(1)-g_{i}(0)}
\end{aligned}
$$

2. Suppose $a+b>2 c$ and $\frac{(1-t) r}{t}>\frac{(a+b-2 c)(a+b)}{(a+b-2 d)(a-b)}$, then the case is exactly the same as (1);

3. Suppose $a+b>2 c$ and $\frac{(1-t) r}{t}=\frac{(a+b-2 c)(a+b)}{(a+b-2 d)(a-b)}$. The equality implies trivial convention $\omega_{i}(C ; C)=\omega_{i}(D ; C)=1$, as the efficiency gain and the fairness loss balances off. The social payoffs are the same regardless playing which strategy. But $a+b>2 c \Rightarrow a>c$, and hence players deviate to $D$ for all $\theta,(C, C)$ cannot be a social equilibrium. 
4. Suppose $a+b>2 c$ and $\frac{(1-t) r}{t}<\frac{(a+b-2 c)(a+b)}{(a+b-2 d)(a-b)}$. The convention is $\omega_{i}(C ; C)=$ $0, \omega_{i}(D ; C)=1$. Given $j$ plays $C, i$ plays $C$ if and only if $c+\theta_{i}\left(g_{i}(0)+h_{i}(0)\right) \geq$ $a+\theta_{i}\left(g_{i}(1)+h_{i}(1)\right)$ which yields a nonempty set $\theta_{i} \geq \frac{a-c}{g_{i}(0)+h_{i}(0)-\left(g_{i}(1)+h_{i}(1)\right)}$ if and only if the interaction effects dominate, i.e. $g_{i}(1)-g_{i}(0)<h_{i}(0)-h_{i}(1)$.

The above yields theorem 2 .

For $(D, D)$ to be a social equilibrium:

The relevant distributional convention is $\omega_{i}(C ; D)$ and $\omega_{i}(D ; D)$. There are three cases:

1. $\omega_{i}(C ; D)=1$ and $\omega_{i}(D ; D)=0: i$ plays $D$ if and only if $d+\theta_{i}\left(g_{i}(0)+h_{i}(0)\right) \geq$ $b+\theta_{i}\left(g_{i}(1)+h_{i}(1)\right)$ if and only if:

(a) $\theta_{i} \geq \frac{b-d}{g_{i}(0)+h_{i}(0)-\left(g_{i}(1)+h_{i}(1)\right)}$ if the interaction effects strictly dominate. The condition is trivially satisfied if $b \leq d$ as is the case in the coordination game and the prisoner's dilemma game;

(b) $\theta_{i} \leq \frac{b-d}{g_{i}(0)+h_{i}(0)-\left(g_{i}(1)+h_{i}(1)\right)}$ if the conformity effects dominate strictly dominate. In this case a necessary condition is $b<d$, which is not possible in the chicken game;

(c) $d \geq b$ if the conformity effects equal to the interaction effects.

2. $\omega_{i}(C ; D)=0$ and $\omega_{i}(D ; D)=1: i$ plays $D$ if and only if $d+\theta_{i}\left(g_{i}(1)+h_{i}(0)\right) \geq$ $b+\theta_{i}\left(g_{i}(0)+h_{i}(0)\right) \Leftrightarrow \theta_{i} \geq \frac{b-d}{g_{i}(1)-g_{i}(0)}$ which is again trivially satisfied when $b \leq d$ as is the case in the coordination game and the prisoner's dilemma game;

3. Trivial convention $\omega_{i}(C ; D)=\omega_{i}(D ; D)=1$ : $i$ plays $D$ if and only if $d \geq b$.

For $(C, D)$ to be a social equilibrium (the case for $(D, C)$ is symmetric): there are three cases to consider 2930

1. $\omega_{i}(C ; C)=1, \omega_{i}(C ; D)=1$. The equilibrium conditions are $b+\theta_{1}\left(g_{1}(1)+\right.$ $\left.h_{1}(1)\right) \geq d+\theta_{1}\left(g_{1}(0)+h_{1}(0)\right) \Leftrightarrow \theta_{1} \leq \frac{b-d}{g_{1}(0)+h_{1}(0)-\left(g_{1}(1)+h_{1}(1)\right)}$ if the interaction effects strictly dominate and for all $\theta_{1}$ otherwise; and $a+\theta_{2}\left(g_{2}(0)+h_{2}(0)\right) \geq$ $c+\theta_{2}\left(g_{2}(1)+h_{2}(0)\right) \Leftrightarrow \theta_{2} \leq \frac{a-c}{g_{2}(1)-g_{2}(0)} ;$

2. $\omega_{i}(C ; C)=1, \omega_{i}(C ; D)=0$. The equilibrium conditions are: $b+\theta_{1}\left(g_{1}(0)+\right.$ $\left.h_{1}(0)\right) \geq d+\theta_{1}\left(g_{1}(1)+h_{1}(1)\right) \Leftrightarrow \theta_{1} \leq \frac{b-d}{g_{1}(0)+h_{1}(0)-\left(g_{1}(1)+h_{1}(1)\right)}$ if the conformity effects strictly dominate and for all $\theta_{1}$ otherwise; and $a+\theta_{2}\left(g_{2}(0)+h_{2}(0)\right) \geq$ $c+\theta_{2}\left(g_{2}(1)+h_{2}(1)\right) \Leftrightarrow \theta_{2} \leq \frac{c-a}{g_{2}(0)+h_{2}(0)-\left(g_{2}(1)+h_{2}(1)\right)}$ if the conformity effects strictly dominate and for all $\theta_{2}$ otherwise;

\footnotetext{
${ }^{29}$ For ease of exposition, I omit discussions of cases of trivial convention or where the interaction effects exactly offset conformity effects.

${ }^{30}$ Since $c>d$, it is not possible to have $\omega_{i}(D ; D)=1$ but $\omega_{i}(C ; C)=0$.
} 
3. $\omega_{i}(C ; C)=0, \omega_{i}(C ; D)=1$. Note such convention only occurs when $a>c, b>$ $d$. The equilibrium conditions are: $b+\theta_{1}\left(g_{1}(1)+h_{1}(0)\right) \geq d+\theta_{1}\left(g_{1}(0)+h_{1}(0)\right)$ which holds for all $\theta_{1}$; and $a+\theta_{2}\left(g_{2}(1)+h_{2}(0)\right) \geq c+\theta_{2}\left(g_{2}(0)+h_{2}(0)\right)$ which

also holds for all $\theta_{2}$. otherwise.

Theorem 3 follows from the above discussions.

\subsection{The numerical example of public-good games.}

The relevant social indices are:

$$
\begin{gathered}
\omega_{1}^{S, B}\left(c_{1} ; 0\right)=\frac{-c_{1}+14 \ln \left(c_{1}+1\right)}{-13+14 \ln (14)} \\
\omega_{2}^{S}\left(c_{2} ; c_{1}\right)= \begin{cases}\frac{-c_{2}+14 \ln \frac{c_{1}+c_{2}+1}{c_{1}+1}}{c_{1}-13+14 \ln \frac{14}{c_{1}+1}} & \text { if } 5.3 \geq c_{1}>0 \\
\frac{20-c_{2}+14 \ln \frac{c_{1}+c_{2}+1}{c_{1}+21}}{7+c_{1}+14 \ln \frac{14}{c_{1}+21}} & \text { if } c_{1}>5.3\end{cases}
\end{gathered}
$$

The cut-off value of 5.3 is obtained by solving for $c_{1}$ in the equation $\pi_{1}^{S}\left(c_{1}, 0\right)+$ $\pi_{2}^{S}\left(c_{1}, 0\right)=\pi_{1}^{S}\left(c_{1}, 20\right)+\pi_{2}^{S}\left(c_{1}, 20\right)$, since for small $c_{1}$, contributing 0 induces the least efficient social outcome, while for large $c_{2}$, contributing 20 induces the least efficient social outcome.

$$
\text { In the best-shot game, let } \bar{c}_{2}\left(c_{1}\right)=\max \left\{c_{2}: c_{2} \in \arg \max _{c_{2} \in[0,20]} \pi_{1}^{B}\left(c_{1}, c_{2}\right)+\pi_{2}^{B}\left(c_{1}, c_{2}\right)\right\} \text {. }
$$

$\bar{c}_{2}$ is non-increasing in $c_{1}$. Numerical search yields that $\bar{c}_{2}(4.8)=0$. That is, for $c_{1} \geq 4.8$, it is socially efficient for 2 to free ride. Since the interaction effects dominate conformity effects in the social payoffs, any $c_{2}>c_{1}$ is dominated by $c_{2}=0$. So for $c_{1} \geq 4.8$, the relevant strategies for 2 are $c_{2} \leq c_{1}$, the social indices for which are given by

$$
\omega_{2}^{B}\left(c_{2} ; c_{1}\right)=\frac{c_{1}-c_{2}}{c_{1}} \text { for } c_{2} \leq c_{1} \text { and } c_{1} \geq 4.8
$$

Now I examine the conditions for $\left(c_{1}, 0\right)$ to be a social equilibrium in the two games.

(1) In the summation game:

1's total payoff of playing strategy $c_{1}$ under the first-order belief of 0 and 
second-order belief of $c_{1}^{*}$ is:

$$
u_{1}^{S}\left(c_{1}, 0, c_{1}^{*}\right)=\left\{\begin{array}{cl}
20-c_{1}+10 \ln \left(c_{1}+1\right)+\theta_{1}\left[-\left(\frac{-c_{1}+14 \ln \left(c_{1}+1\right)}{-13+14 \ln (14)}-1\right)^{2}\right. & \\
\left.-4 \frac{-c_{1}+14 \ln \left(c_{1}+1\right)}{-13+14 \ln (14)}\right] & \text { if } 5.3 \geq c_{1}^{*}>0 \\
20-c_{1}+10 \ln \left(c_{1}+1\right)+\theta_{1}\left[-\left(\frac{-c_{1}+14 \ln \left(c_{1}+1\right)}{-13+14 \ln (14)}-1\right)^{2}\right. & \\
\left.-4 \max \left\{\frac{-c_{1}+14 \ln \left(c_{1}+1\right)}{-13+14 \ln (14)}-\frac{20+14 \ln \frac{c_{1}^{*}+1}{c_{1}^{*}+21}}{7+c_{1}^{*}+14 \ln \frac{14}{c_{1}^{*}+21}}, 0\right\}\right] & \text { if } c_{1}^{*}>5.3
\end{array}\right.
$$

2 's total payoffs of playing strategy $c_{2}$ under the first-order belief of $c_{1}^{*}$ and second-order belief of 0 is:

$$
u_{2}^{S}\left(c_{2}, c_{1}^{*}, 0\right)=\left\{\begin{array}{cc}
20-c_{2}+4 \ln \left(c_{1}^{*}+c_{2}+1\right)+\theta_{2}\left\{-\left[\frac{-c_{2}+14 \ln \frac{c_{1}^{*}+c_{2}+1}{c_{1}^{*}+1}}{c_{1}^{*}-13+14 \ln \frac{14}{c_{1}^{*}+1}}-1\right]^{2}\right. & \\
\left.-4\left[\max \left\{\frac{-c_{2}+14 \ln \frac{c_{1}^{*}+c_{2}+1}{c_{1}^{*}+1}}{c_{1}^{*}-13+14 \ln \frac{14}{c_{1}^{*}+1}}-\frac{-c_{1}^{*}+14 \ln \left(c_{1}^{*}+1\right)}{-13+14 \ln (14)}, 0\right\}\right]\right\} & \text { if } 5.3 \geq c_{1}^{*} \geq 0 \\
20-c_{2}+4 \ln \left(c_{1}^{*}+c_{2}+1\right)+\theta_{2}\left\{-\left[\frac{20-c_{2}+14 \ln \frac{c_{1}^{*}+c_{2}+1}{c_{1}^{*}+21}}{7+c_{1}^{*}+14 \ln \frac{14}{c_{1}^{*}+21}}-1\right]^{2}\right. & \\
\left.-4\left[\max \left\{\frac{20-c_{2}+14 \ln \frac{c_{1}^{*}+c_{2}+1}{c_{1}^{*}+21}}{7+c_{1}^{*}+14 \ln \frac{14}{c_{1}^{*}+21}}-\frac{-c_{1}^{*}+14 \ln \left(c_{1}^{*}+1\right)}{-13+14 \ln (14)}, 0\right\}\right]\right\} & \text { if } c_{1}^{*}>5.3
\end{array}\right.
$$

(2) In the best-shot game:

1's total payoff of playing strategy $c_{1}$ under the first-order belief of 0 and second-order belief of $c_{1}^{*} \geq 4.8$ is:

$$
u_{1}^{B}\left(c_{1}, 0, c_{1}^{*}\right)=20-c_{1}+10 \ln \left(c_{1}+1\right)+\theta_{1}\left[-\left(\frac{-c_{1}+14 \ln \left(c_{1}+1\right)}{-13+14 \ln (14)}-1\right)^{2}\right]
$$

Note there are no interaction effects. On the other hand, given the first-order belief of $c_{1}^{*} \geq 4.8$ and second-order belief of 0,2 's total payoff of playing strategy $c_{2} \leq c_{1}^{*}$ is:

$u_{2}^{B}\left(c_{2}, c_{1}^{*}, 0\right)=20-c_{2}+4 \ln \left(c_{1}^{*}+1\right)+\theta_{2}\left\{-\left[\frac{c_{1}^{*}-c_{2}}{c_{1}^{*}}-1\right]^{2}-4\left[\max \left\{\frac{c_{1}^{*}-c_{2}}{c_{1}^{*}}-\frac{-c_{1}^{*}+14 \ln \left(c_{1}^{*}+1\right)}{-13+14 \ln (14)}, 0\right\}\right]\right.$

Figure 3 lists 1's optimal contribution $c_{1}^{*}$ given first-order belief of 0 and secondorder belief of $c_{1}^{*}$ in the two games 31 Figure 4 lists the upper bound of $\theta_{2}$ for 2 to prefer to free ride given first-order belief of $c_{1}^{*} \geq 3$ and second-order belief of $0{ }^{32}$

\footnotetext{
${ }^{31}$ As discussed above, $c_{1}^{*}>0$ in the summation game and $c_{1}^{*} \geq 4.8$ in the best-shot game.

${ }^{32}$ This is a continuous game, while in experiments, subjects typically can only make integer contributions. Thus with an eye to match the experimental results, I say 2 prefers to free ride as long as the optimal $c_{2}<.5$.
} 


\begin{tabular}{|c|c|c|}
\hline$\theta_{1}$ & $c_{1}^{*}=\arg \max _{c_{1} \in[0,20]} u_{1}^{S}\left(c_{1}, 0, c_{1}^{*}\right)$ & $c_{1}^{*}=\arg \max _{c_{1} \in[0,20]} u_{1}^{B}\left(c_{1}, 0, c_{1}^{*}\right)$ \\
\hline 0 & 9 & 9 \\
1 & 8.22 & 9.01 \\
2 & 7.1 & 9.02 \\
3 & 5.49 & 9.03 \\
4 & 3.5 & 9.04 \\
4.5 & 2.59 & 9.04 \\
5 & 1.85 & 9.05 \\
6 & 0.89 & 9.06 \\
7 & 0.37 & 9.07 \\
8 & 0.06 & 9.08 \\
9 & 0 & 9.08 \\
10 & 0 & 9.09 \\
\hline
\end{tabular}

Figure 3: 1's optimal contribution under beliefs $\left(0, c_{1}^{*}\right)$ in the two games

\begin{tabular}{|c|c|c|c|c|}
\hline$c_{1}^{*}$ & $\theta_{2}^{*}=\sup$ & $\theta_{2}: 0=\arg \max _{c_{2} \in[0,20]} u_{2}^{S}\left(c_{2}, c_{1}^{*}, 0\right)$ & $\theta_{2}^{*}=\sup$ & $\theta_{2}: 0=\arg \max _{c_{2} \in[0,20]} u_{2}^{B}\left(c_{2}, c_{1}^{*}, 0\right)$ \\
\hline 3 & & 0.23 & & $\infty$ \\
\hline 4 & & 0.56 & & $\infty$ \\
\hline 5 & & 0.77 & & $\infty$ \\
\hline 6 & & 1.74 & & $\infty$ \\
\hline 7 & & 5.1 & & $\infty$ \\
\hline 8 & & 15.2 & & $\infty$ \\
\hline 9 & & 50 & & $\infty$ \\
\hline
\end{tabular}

Figure 4: The upper bound of $\theta_{2}$ for 2 to prefer to free ride given beliefs $\left(c_{1}^{*}, 0\right)$

Finally I examine under what circumstances can $(0,0)$ be a social equilibrium. Given the opponent contributes 0 , the social implications of i's action are the same in both games:

$$
u_{i}^{S, B}\left(c_{i}, 0\right)=20-c_{i}+p_{i} \ln \left(c_{i}+1\right)+\theta_{2}\left(-\left[\frac{-c_{i}+14 \ln \left(c_{i}+1\right)}{-13+14 \ln 14}-1\right]^{2}-4 \frac{-c_{i}+14 \ln \left(c_{i}+1\right)}{-13+14 \ln 14}\right)
$$

Numerical search for values of $\theta_{i}$ such that 0 maximizes $u_{i}\left(c_{i}, 0\right)$ yields $\theta_{1} \geq$ $6.7, \theta_{2} \geq 1.97$. 


\section{References}

Andreoni, James, "Impure Altruism and Donations To Public Goods: A Theory of Warm-Glow Giving," The Economic Journal, 1990, 100, 464-477.

- and John H. Miller, "Giving According to GARP: An Experimental Test of the Consistency of Preferences for Altruism," Econometrica, 2002, 70 (2), 737-753.

- , Paul M. Brown, and Lise Vesterlund, "What Produces Fairness? Some Experimental Evidence," Games and Economic Behavior, 2002, 40, 1-24.

Bolton, Gary and Axel Ockenfels, "ERC: A Theory of Equity, Reciprocity, and Competition," American Economic Review, 2000, pp. 166-193.

Brandts, Jordi and Carles Sola, "Reference Points and Negative Reciprocity in Simple Sequential Games," Games and Economic Behavior, 2001, 36 (2).

Camerer, Colin, Behavioral Game Theory: Experiments on Strategic Interaction, Princeton University Press, 2003.

- and Richard H. Thaler, "Anomalies: Ultamatum, Dictators and Manners," Journal of Economic Perspectives, 1995, 9 (2), 209-219.

Charness, Gary and Matthew Rabin, "Understanding Social Preferences with Simple Tests," Quarterly Journal of Economics, 2002, 117, 817-869.

Cooper, Russel, Douglas V. DeJong, Robert Forsythe, and Thomas W. Ross, "Cooperation without Reputation: Experimental Evidence from Prisoner's Dilemma Games," Games and Economic Behavior, 1996, 12 (2), 187-218.

Dawes, Robyn M. and Richard Thaler, "Anomalies: Cooperation," The Journal of Economic Perspectives, 1988, 2 (3), 187-197.

Dufwenberg, Martin and Georg Kirchsteiger, "A Theory of Sequential Reciprocity," Games and Economic Behavior, 2004, 47, 268-298.

Falk, Armin and Urs Fischbacher, "A Theory of Reciprocity," 2000. forthcoming, Games and Economic Behavior.

- , Ernst Fehr, and Urs Fischbacher, "Testing Theories of Fairness - Intentions Matter," 2000. Working paper, University of Zurich.

Fehr, Ernst and Klaus M. Schmidt, "A Theory of Fairness, Competition, and Cooperation," Quarterly Journal of Economics, 1999, 114, 817 - 868.

Geanakoplos, John, David Pearce, and Ennio Stacchetti, "Psychological Games and Sequential Rationality," Games and Economic Behabior, 1989, 1, 60-79.

Georee, Jacob K. and Charles A. Holt, "Ten Little Treasures of Game Theory and Ten Intuitive Contradictions," American Economic Review, 2001, 91 (5), 1402-1422.

Harrison, Glenn W. and Jack Hirshleifer, "An Experimental Evaluation of Weakest Link/Best Shot Models of Public Goods," Journal of Political Economy, 1989, 97, 201-225.

Kahneman, Daniel and Amos Tversky, eds, Choices, Values, and Frames, 
Cambridge University Press, 2000.

- , Jack L. Knetsch, and Richard H. Thaler, "Fairness and the Assumptions of Economics," Journal of Business, 1986, 59 (4), 285-300.

Prasnikar, Vesna and Alvin E. Roth, "Considerations of Fairness and Strategy: Experimental Data From Sequential Games," The Quarterly Journal of Economics, 1992, 107 (3), 865-888.

Rabin, Matthew, "Incorporating Fairness into Game Theory and Economics," American Economic Review, 1993, 83 (5), 1281-1301.

Sobel, Joel, "Interdependent Preferences and Reciprocity," 2004. Working paper, University of California at San Diego.

Thaler, Richard H., The Winner's Curse: Paradoxes and Anomalies of Economic Life, The Free Press, 1988.

Weibull, Jörgen, "Testing Game Theory," 2004. Forthcoming in "Advances in Understanding Strategic Behaviour: Game Theory, Experiments, and Bounded Rationality: Essays in Honour of Werner Güth", Palgrave. 